\section{Combinatorial Methods Used to Identify New Compositions of Ferromagnetic Shape-Memory Alloys}

Combinatorial materials science is used to systematically study materials properties as a function of composition using a number of small samples differing slightly in composition. In a study published in the March issue of Nature Materials, I. Takeuchi and colleagues from the University of Maryland, Rowan University, and Neocera, Inc. have used thin-film composition spreads of multiferroic Ni$\mathrm{Mn}-\mathrm{Ga}$ alloys, of which the so-called Heusler composition, $\mathrm{Ni}_{2} \mathrm{MnGa}$, is a wellknown shape-memory alloy, to study both the mechanical behavior and ferromagnetic properties of the alloys.

Multiferroic alloys exhibit the ability to switch between two conditions, such as ferromagnetic and paramagnetic, in more than one combination of conditions. The $\mathrm{Ni}-\mathrm{Mn}-\mathrm{Ga}$ alloys are ferromagnetic and are also shape-memory alloys, so, in addition to exhibiting martensitic transitions due to temperature change, shape-memory effects can, in many cases, be induced by an applied field, leading to a number of potential applications. The objective of the study was to determine which compositions of the ternary Ni-Mn-Ga system other than the Heusler composition exhibit ferromagnetic shape-memory effects. This required the development of techniques to measure both mechanical and magnetic properties of a series of compositions of the alloy system.

For measuring magnetization, the researchers used a spread of patterned combinatorial array of $2 \mathrm{~mm} \times 2 \mathrm{~mm}$ square grids and a scanning superconducting quantum interference device (SQUID) microscope. For detecting structural martensitic phase transition, a spread of compositions was deposited as thin films on a series of silicon cantilevers. When the cantilevers are heated causing a phase transformation, they bend, which is detected during visual inspection of the image of the cantilever arrays as a change in reflection resulting from a changing radius of curvature.

Results showed that the strongest magnetization and the highest phase-transition temperatures were observed in regions away from the traditional Heusler composition. However, higher magnetization was coupled with a lower transition temperature. Thus there is a tradeoff between the two properties in the system. It was found that in a ternary system, the Heusler composition does not necessarily yield optimized functionality.
More importantly, this study demonstrates the use of combinatorial methods in conjunction with unique characterization techniques to systematically study an alloy system.

Gopal RaO

\section{Rupture of Rhodamine-Labeled DNA Studied by Simultaneous, Spatially Coincident Optical Trapping and Single-Molecule Fluorescence}

Heretofore, optical trapping and singlemolecule fluorescence, two important techniques in single-molecule research, have not been successfully combined in a single experiment because the light emitted by a single fluorophore is 10 orders of magnitude lower than the light intensity of an optical trap. (Optical traps, also known as optical tweezers, use photon momentum transfer to grasp and manipulate microscopic dielectric particles, such as microspheres, cells, and cellular organelles.) In previous attempts, either the optical trap was separated from the fluorescence region by several millimeters or the two techniques were employed sequentially. Recently, a team of researchers from Stanford University's Biological Sciences, Applied Physics, and Physics departments demonstrated simultaneous, spatially coincident optical trapping and single-molecule fluorescence.

As reported in the February 24 issue of the Journal of Biology, Stanford researcher S.M. Block and co-workers observed changes in single-molecule fluorescence and force-induced strand separation in dye-labeled double-stranded DNA. The researchers attached one end of a 1,010 base-pair DNA duplex to a polystyrene bead with a diameter of $500 \mathrm{~nm}$. The distal end of the DNA, designed with an overhanging segment on the $5^{\prime}$ end, was annealed to a complementary 15 base-pair oligonucleotide, which was anchored on its $5^{\prime}$ end to a cover glass surface (see figure). Tetramethylrhodamine (TAMRA) dyes conjugated to nucleotides were attached to complementary bases on the free end of the DNA, that is, on the 3 ' end of the 15-mer and on the 5 end of the long DNA duplex. In such close proximity, fluorescence of the dye molecules is self-quenched. The researchers note that the very short distances probed by TAMRA self-quenching (about $12 \mathrm{~nm}$ ) is smaller, in most cases, than those probed by fluorescence-resonance energy transfer.

The instrument developed by the researchers incorporates a microscope, a laser for trapping $(1064 \mathrm{~nm})$, another to detect position $(827 \mathrm{~nm})$, and a third for fluorescence excitation (514 nm). By trap- ping the DNA-bead complex and moving the microscope stage at constant velocity, the researchers pulled the bead from the center of the trap and increased the load until rupture occurred, that is, until the 15 base-pair duplex "unzipped" into two single strands. Before rupture, the researchers observed low light levels, indicative of the quenched dye state, and a monotonic increase in the force. At rupture, the force abruptly decreased from $9 \mathrm{pN}$ to $0 \mathrm{pN}$ and the light level increased, indicating that the two fluorophores separated, thereby unquenching the dye bound to the 15-mer. Two control experiments with single dye molecules, in which TAMRA was bound either to the long DNA duplex or to the 15-mer, demonstrated that rupture corresponds to strand separation and not to breakage of the linkage holding the DNA to the cover slip.

The researchers believe that simultaneous optical trapping and single-molecule fluorescence will supply vital information about the sequence of molecular events. Furthermore, the researchers anticipate that "this technique will have broad appli-
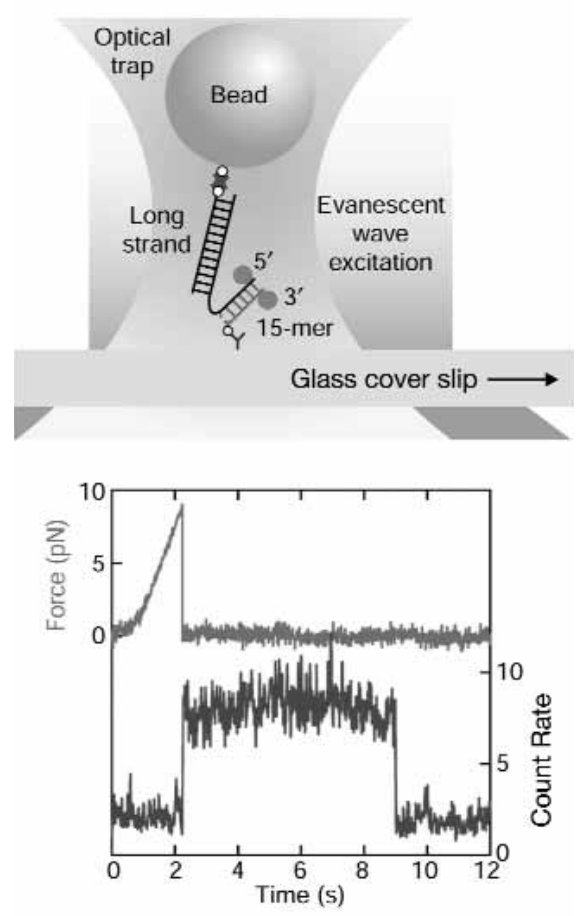

Figure. A combined optical trapping and fluorescence experiment to unzip DNA. (a) Schematic of the experimental geometry; (b) simultaneous measurements of the force required to rupture (top trace) and the fluorescence (bottom trace). The dye unquenched upon rupture and then later bleached. Courtesy Journal of Biology 2 (6)(2003); http://jbiol.com/content/2/1/6. 
cability to the study of fundamental biological questions, by providing a direct means to study coupling between biochemical and biomechanical reaction cycles."

STEVEN TROHALAKI

\section{High-Velocity Ballistic Impact with Boron Carbide Produces Localized Amorphization}

Localized amorphization induced by shock has been observed in crystalline boron carbide. Mingwei Chen and Kevin Hemker of Johns Hopkins University and James McCauley of the U.S. Army Research Laboratory at Aberdeen Proving Ground have demonstrated that extremely high-rate deformation can initiate formation of amorphized bands in the rhombohedral crystal structure of $\mathrm{B}_{4} \mathrm{C}$.

Boron carbide is commonly used as ballistic armor. Although the material is effective for protection against low-energy projectiles such as bullets from handguns, it is less effective upon more powerful impacts. As reported in the March 7 issue of Science, Chen, McCauley, and Hemker found that the measured drop in the impact resistance of boron carbide at impact pressures in the range of $20-23 \mathrm{GPa}$ could be attributed to the formation of nanoscale intragranular amorphous bands in the crystal.

The researchers observed the amorphous bands by high-resolution electron microscopy (HREM). They used transmission electron microscope electron energy-loss spectroscopy to compare the chemical composition of the amorphous regions to that of the crystalline $B_{4} C$. These spectra allowed the researchers to rule out the possibility of a pressureinduced decomposition or chemical reaction since there was no detectable difference in chemical composition. The researchers also determined the HREM images were not consistent with rebonding of two cracked surfaces or melting. Accordingly, they believe that the amorphization is a solid-state transformation instigated by the ballistic event.

Recent diamond anvil studies demonstrated a phase transition at a pressure around $20 \mathrm{GPa}$, and previous nanoindentation experiments at even higher pressures suggested amorphization, but this speculation was discounted based on Raman spectra. The researchers in this study suggest that their results warrant renewed scrutiny of the previous interpretations of the diamond anvil and nanoindentation results.

The drop in performance of boron carbide when exposed to high-impact pressures has been known for years. The cur- rent research provides a microscopic explanation for this highly unfavorable property for a material employed as ballistic armor. The researchers do not propose a means to avoid formation of the amorphization regions that appear to be responsible for the drop in strength of crystalline boron carbide. Nevertheless, they speculate that this improved understanding of how shock can alter materials properties and permit the synthesis of novel structures could further expand the realm of possibilities for innovative materials synthesis.

Chen said, "It's like having a sturdy table and suddenly kicking the legs out from underneath it."

"This discovery was very enlightening, because it tells us that under extremely high pressures the crystal structure collapses and forms these nanoscale amorphous bands," said Hemker. "Then the material fractures along these bands because the glassy material appears to be weaker than the crystalline boron carbide."

McCauley said, "The question now is, how should we try to change the boron carbide? We intend to try modifying the material's grain structure, its chemistry, and the additives used in making it. The goal will be to have the amorphization occur at higher impact pressures. Then the armor would provide better protection against a wider range of threats."

Although the findings have immediate implications in the production of improved armor materials, the researchers said that their observations also provide experimental evidence that extreme conditions in pressure, temperature, and/or loading and quenching rates can lead to the creation of entirely new materials or structures with substantially altered physical and mechanical properties.

"We have a new appreciation for how localized loss of crystalline structure can substantially alter materials properties," Hemker said. "I anticipate this understanding will allow scientists and engineers to tailor boron carbide's properties in a way that has not been exploited to date. We do not need to rely on chemical modifications or phase transformations to pursue novel materials properties. I hope our research will help to motivate exciting new avenues in materials design."

EMILY JARVIS

\section{Carbon Nanofibers with Smaller Diameter Rather than Larger Promote More Osteoblast Functions}

Nanomaterials in the form of ceramics, polymers, metals, and carbon nanofibers have recently been shown to improve bone, vascular, bladder, cartilage, and neural cell function. Doctoral student Rachel Price, under the direction of Thomas J. Webster in the Biomedical Engineering Department at Purdue University, has demonstrated that smallerdiameter carbon nanofibers promote more functions of the osteoblast, the boneforming cell, than do nanofibers with larger diameters. Such a conclusion was based on evidence that a statistically larger number of osteoblasts adhered, proliferated, and deposited calcium-containing material on the smaller-diameter (60-nm) carbonfiber compacts than to the larger-diameter (125-nm) carbon-fiber compacts, or to either a cobalt chromium or a titanium alloy. The latter two materials are currently used in dental and orthopedic applications.

As reported in the May issue of Biomaterials (24:11), there was a $33 \%$ increase in the number of adherent osteoblasts on the smaller carbon fibers than the larger and up to an $800 \%$ increase over the titanium alloy. In addition, in a carbon nanofiber/polycarbonate urethane composite, osteoblast adhesion increased on those composites when higher ratios of carbon nanofibers were incorporated.

Price said, "Another interesting finding is that competitive cell functions are adversely affected on the smaller carbon fibers over the same time span. Increased osteoblast function and decreased competitive cell functions imply that better bonding to juxtaposed bone can be achieved through the use of carbon nanofibers."

\section{Novel Method for Control of Quantum Entanglement Developed}

Entanglement refers to a quantum phenomenon whereby measurements performed on one particle affect another particle that is far away. Quantum entanglement can be exploited in quantum information applications such as cryptography and quantum computation. A recent report by Roberto Merlin and coworkers in the Departments of Physics at the University of Michigan, Ann Arbor, and the University of Notre Dame describes the controlled generation of entangled states involving the spin of $\mathrm{Mn}^{2+}$ and donor impurities in CdTe quantum wells. Their procedure relies on photoexcited excitons to mediate the interaction between spins. The method allows a previously unavailable level of control of particle entanglement. According to Merlin, "the procedure is potentially set-specific and scalable, thereby holding promise for quantum computing applications."

As described in the March issue of 